\title{
Two-Photon Absorption (TPA) Backside Pulsed Laser Tests in the LM324
}

\author{
I. López-Calle, F. J. Franco, J. G. Izquierdo and J. A. Agapito
}

\begin{abstract}
Experiments to obtain XY scans on the surface of an amplifier at different depths and energy values were performed at the UCM, the results of which are shown and discussed in this paper.
\end{abstract}

Index Terms - Laser, LM324, Operational Amplifiers, Single Event Transients, Two-Photon Tests.

\section{INTRODUCTION}

$\mathrm{A}$ NALOG single event transients induced by laser have been studied in typical devices such as operational amplifiers, regulators, comparators, etc. Usually, experiments consist in scanning the front surface to find the most sensitive devices [1]-[3] or selecting one interesting device and exploring its sensitivity along the Z-axis [4]. In this paper, both kinds of test are mixed using Two-Photon Absorption (TPA) [5] in order to perform XY scans at different focus depth. Also, these scans were done changing the laser energy. The final objective of this research line is to obtain the laser parameters configuration of two photon irradiation to emulate the behavior of the LM324 amplifier under ion hit. These results show that we can easily choose the laser parameters and the depth at the focus from the backside to emulate any ion hit. In future works we could match the laser parameters and the ion LET by means of Akkerman \& Barack experimental relationship [6] as a function of the mass and the energy of the ion. Thus, this experimental set-up will be used to evaluate the quality of our model.

\section{EXPERIMENTAL SET-UP}

The experiments were performed at the UCM Multiphotonic Spectroscopy and Femtosecond facility using a Ti:sapphire laser followed by a regenerative amplifier. The

This work was supported in part by the EMULASER project (CDTI/PNE034/2006) and by the Spanish MEC through GrantCTQ2008-02578/BQU, and Consolider SAUUL CSD2007-00013. Finally, I. López-Calle gratefully acknowledges the grant offered by the "Miguel Casado San José" private foundation.

I. López-Calle, F. J. Franco and J. A. Agapito are with the Departamento de Física Aplicada III, Facultad de Físicas, Universidad Complutense de Madrid (UCM), 28040 Madrid (Spain) (e-mail: isabelcalle, monti, agapito@fis.ucm.es).

J. G. Izquierdo is with the Servicio de Espectroscopía Multifotónica y de Femtosegundo, CAI de Espectroscopía, Facultad de Químicas, Universidad Complutense de Madrid (UCM), 28040 Madrid (Spain) (e-mail: jegonzal@quim.ucm.es). laser wavelength is tunable between $300-3000 \mathrm{~nm}$. For twophoton absorption processes in silicon, 80 -fs laser pulses at a frequency of $1 \mathrm{kHz}$ and a wavelength of $1300 \mathrm{~nm}$ was fixed. The energy was measured with a typical commercial power meter.

The device was mounted on a motorized $x y z$ stage with 0.1 $\mu \mathrm{m}$ resolution and it could be observed with a CCD camera to allow the correct placement of the laser. Laser beam was focused with a 50x long working-distance microscope objective, appropriate for infrared light and making the spot diameter on the order of $1.5 \mu \mathrm{m}$.

The amplifiers were biased as buffers with an input voltage of $4.0 \mathrm{~V}$ and bipolar power supplies of $\pm 15 \mathrm{~V}$. The outputs were connected to a digital oscilloscope with $8 \mathrm{pF}$ probes. This device was triggered by means of an external signal coming from the laser. Fig. 1 shows a graphical description of the laser system.

A specific LabView application was developed to control all the devices and to store the data provided by the oscilloscope for a later analysis.

Two National Semiconductor LM324N, both of them belonging to the same batch and in plastic package, were decapsulated frontside and backside respectively. The first one was used to measure and locate all components of the chip and to measure the thickness of the chip $(350 \mu \mathrm{m})$. The second was prepared for backside two photon irradiations. We have pointed out that all layers of the chip can be reached by backside irradiation without previous thinning of the silicon bulk at a wavelength of $1300 \mathrm{~nm}$.

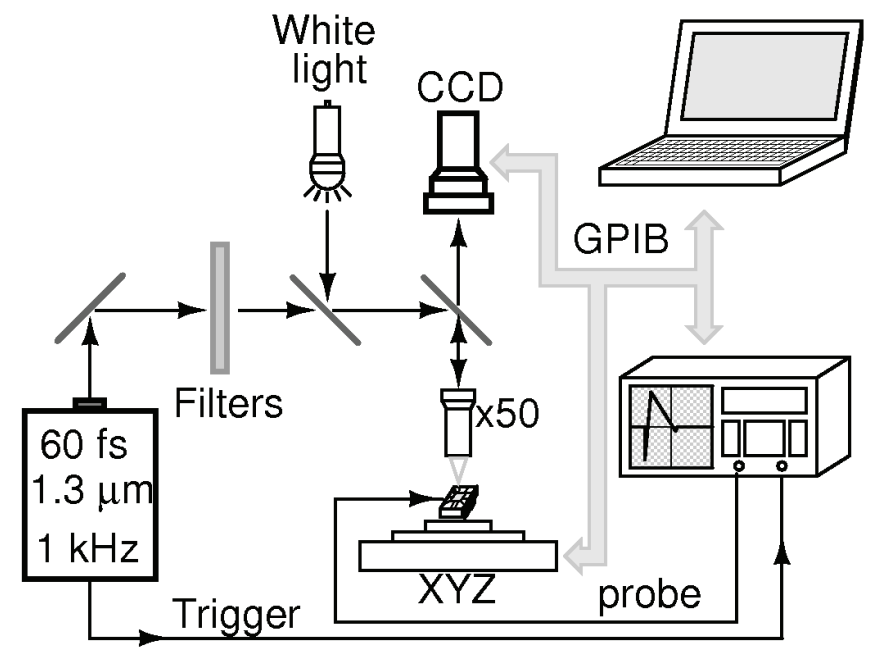

Fig. 1. Laser configuration and test set-up. 


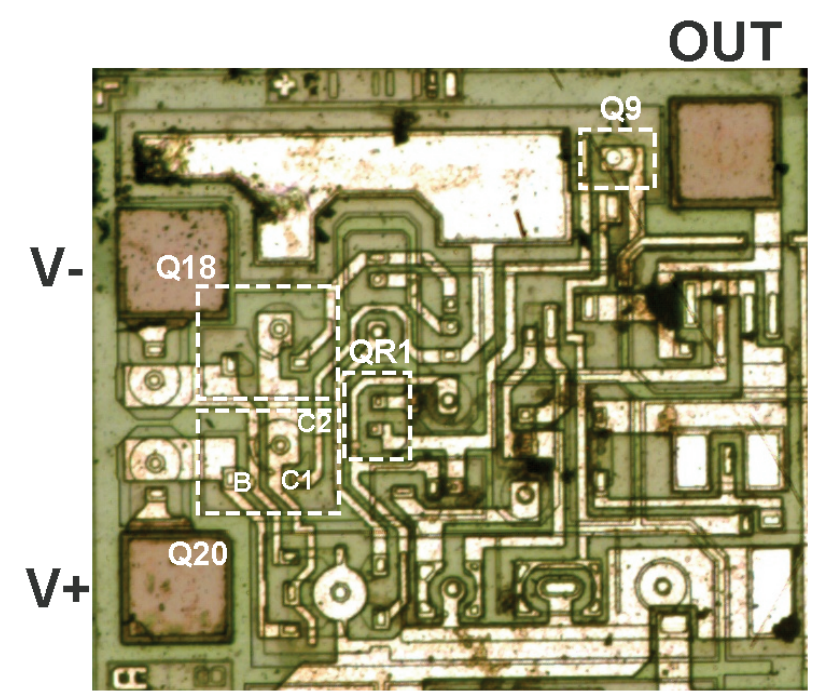

Fig. 2. Microphotograph of the LM124 lay-out. Spots where the laser was focused are marked in white at different sensitive positions.

\section{RESULTS}

First of all a scan was performed at different sensitive positions of Q18, Q20 and Q9 (Fig. 2) for several values of energy and focus depth. Fig. 3 shows that a detectable small transient is detected only for energies higher than $0.12 \mathrm{~nJ}$ at the most sensitive $z$ position of $265 \mu \mathrm{m}$. Fig. 3 also shows that the higher the energy the larger the volume of the sensitive area appears to be.

On the other hand the critical value of the laser energy suitable to produce undesired output transients under non linear absorption is clearly detected in Fig. 4.

Moreover there is a change in the shape of transients as function of the focus depth as well as the laser pulse energy. Fig. 5-6 show the change of the transients for different energy values at the same position.

Furthermore, scans of the whole amplifier have been carried out at a fixed energy of $0.6 \mathrm{~nJ}$. Maps of the sign and the amplitude of the output voltage transients are presented in Fig. 7 and 8 for two different focus depths. A change in the kind of transients is pointed out at transistors Q20 and Q18.

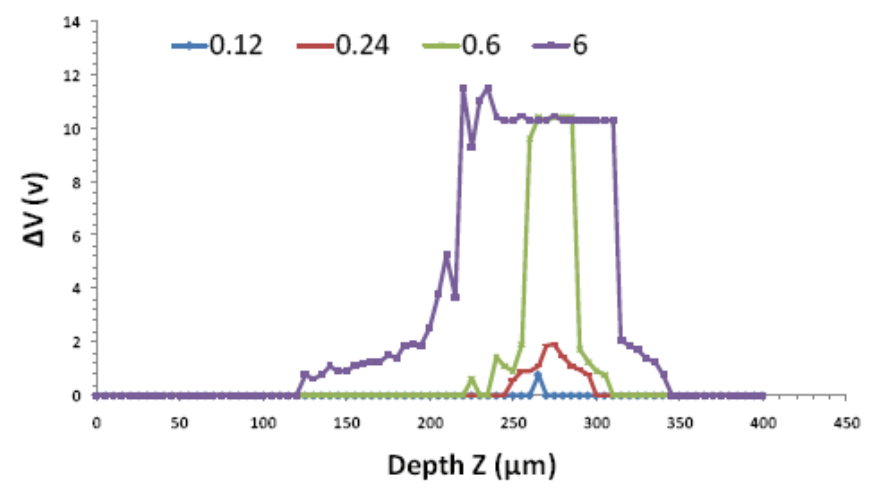

Fig. 3. Two photon induced single-event transients at C1 of transistor Q20. The curves represent the increment of the output signal for different energies of the pulsed laser: from $0.12 \mathrm{~nJ}$ to $6 \mathrm{~nJ}$. The $\mathrm{X}$ axis represents the position of the focus relative to the backside surface of the device in $z=0$.

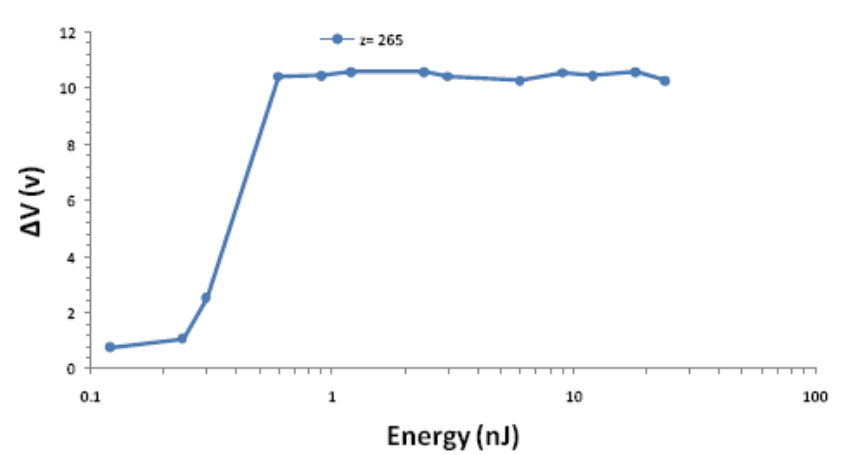

Fig. 4. Two photon induced single-event transients at $\mathrm{C} 1$ of transistor Q20. The curves represent the increment of the output signal for different energies of the pulsed laser: from $0.12 \mathrm{~nJ}$ to $6 \mathrm{~nJ}$. The $\mathrm{X}$ axis represents the position of the focus relative to the backside surface of the device in $\mathrm{z}=0$.

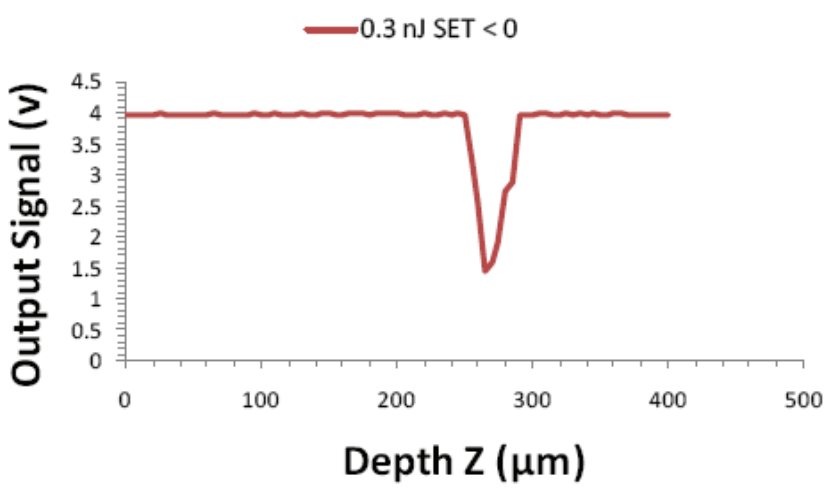

Fig. 5. Output signal transients at $\mathrm{C} 1$ of Q20. Only negative sign at $\mathbf{0 . 3} \mathbf{n J}$

(a)

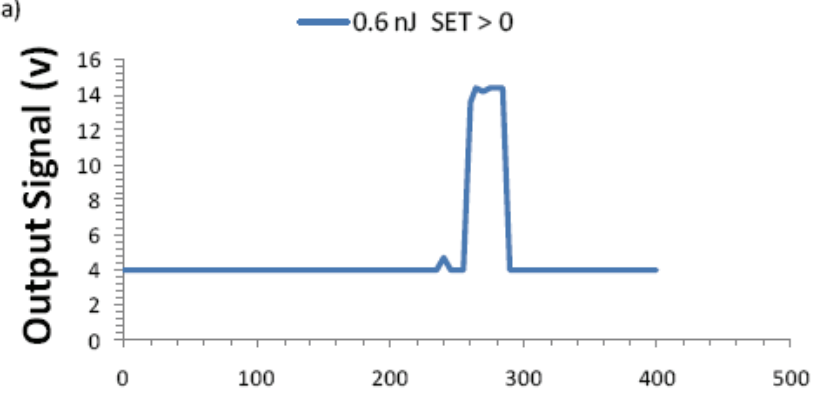

(b)

Depth Z ( $\mu \mathrm{m})$

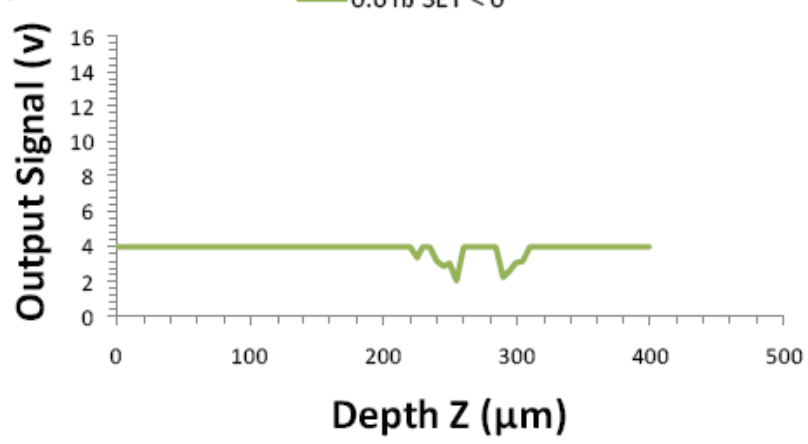

Fig. 6. Output signal transients at $\mathrm{C} 1$ of Q20. a) Only positive sign at $\mathbf{0 . 6} \mathbf{n J}$. b) Negative transients at different $z$ values. 


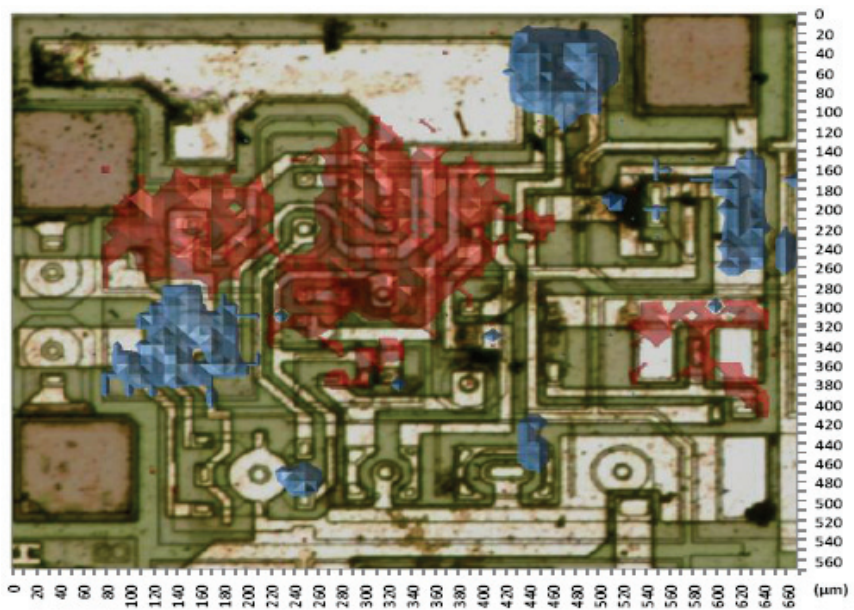

Fig. 7. 2D map showing the sign of the transients at different xy positions. Red color are positive transients. Blue color are negative transients. Focus depth at $\mathbf{3 0 0} \mu \mathrm{m}$. Pulse energy at $0.6 \mathrm{~nJ}$. Full color figures are available in the electronic version of the paper.

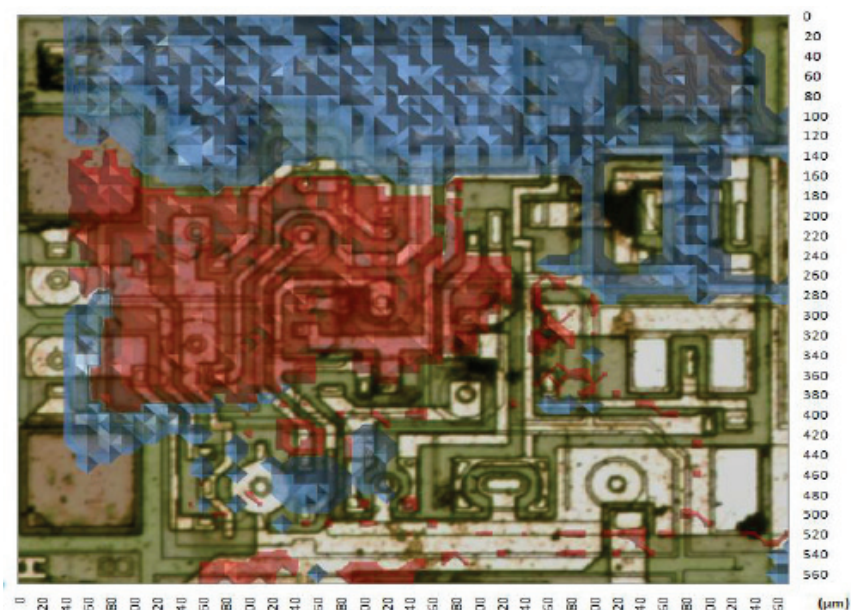

Fig. 8. 2D map showing the sign of the transients at diferents xy positions. Red color are positive transients. Blue color are negative transients. Focus depth at $270 \mu \mathrm{m}$. Pulse energy at $0.6 \mathrm{~nJ}$.

These transistors belong to the differential amplifier inside the input stage as it can be seen in the simplified LM324 schematic (Fig. 9) [7].

Finally a characteristic plot of output peak voltage vs. peak full width at half maximum of the transients is shown in Fig. 10 in accordance to the behavior of the amplifier under ion hit [1]-[3], [8].

Once the previous analysis was done, the goal of the experiments was to obtain XY scans on the whole surface of an amplifier at different depths and with several values of energy by means of optical filters. The range was between 0.6 and 6 $\mathrm{nJ}$. The LM324 being a quad operational amplifier, only one of the four operational amplifiers was tested. The total surface to be scanned was $660 \mu \mathrm{m} \times 560 \mu \mathrm{m}$ with a step of $10 \mu \mathrm{m}$. The scanning rate was one shot for each position and one sensitivity map took approximately two and a half hours. It also must be highlighted that all the Z-values shown in this paper are referenced to the rear surface of the chip and not to the front side.

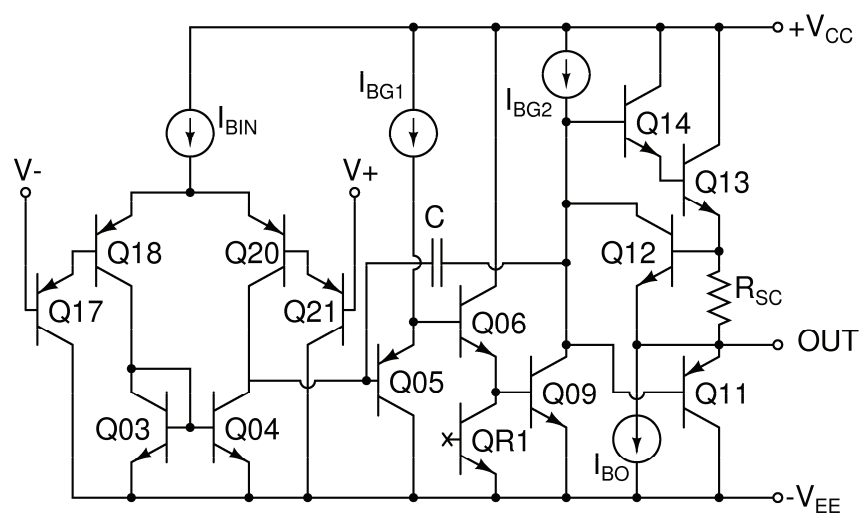

Fig. 9. Simplified LM124 diagram.

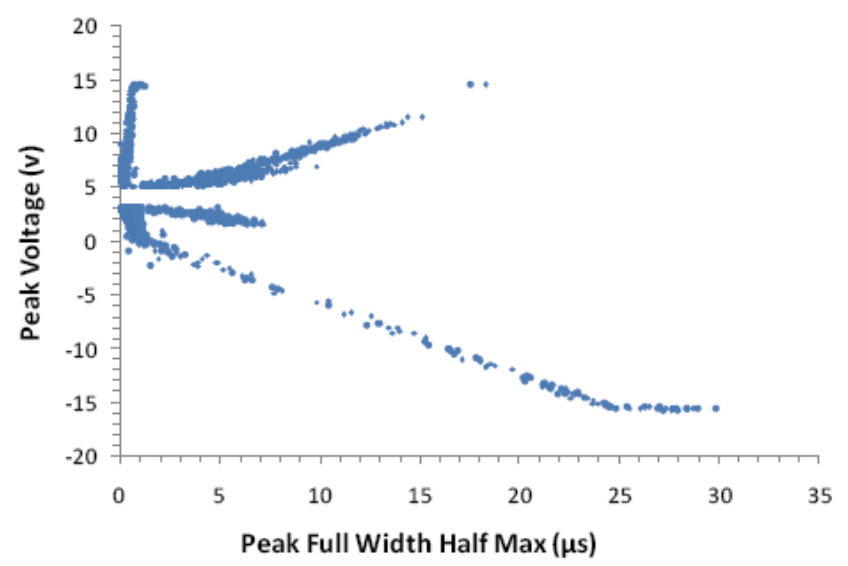

Fig. 10. Peak voltage vs. Pulse width for the LM324 under TPA backside focus depth $300 \mu \mathrm{m}$ and $1.2 \mathrm{~nJ}$.

\section{A. Data Analysis}

Once the scans had been completed, data coming from the oscilloscope and stored in the PC hard disk were checked to evaluate the presence of SETs and their characteristics, such as the increment of the output voltage, duration, polarity, etc. Graphs attached to this paper summarize the results of the experiments. Results are shown using two methods: First, the size of the SET are symbolized using colors on a picture of the device (Fig. $11 \& 12$ left). Also, a 3D-graph shows the variability of the sensitivity to deposited charge in the device given the depth and the energy of the laser (Fig. $11 \& 12$ right). With the purpose of understanding the sensitive areas of the device, 3D graphs were generated. In these pictures one can distinguish at a glance which area of the device is the most sensitive in the performed scans (Fig. 11c)

Data concerning the SET duration as well as its character (positive, negative, bipolar) were also obtained but are not included in this paper given that the provided information is similar to that shown in the others graphs.

\section{DISCUSSION}

Most of the papers found in the literature usually depict the response of the device to a whole surface scan or select a critical spot to be exhaustively studied along the Z-axis. To the authors' knowledge, this is one of the first papers showing XY scans not only in the surface but at different values of depth. 
Thus, buried layers had been detected and fully characterized. E.g., results show that at a depth of $270 \mu \mathrm{m}$ and using $0.6 \mathrm{~nJ}$ almost every part of the devices is laser sensitive generating SETs the amplitude of which is enough to lead the operational amplifier output to the saturation voltage values. However, a shift of around some tens of $\mu \mathrm{m}$ upwards or downwards makes the device to show fewer SETs. In fact, at depth values of $210 \& 300 \mu \mathrm{m}$ only two well known internal devices (Q09, QR1) are SET sensitive [5].

Another interesting point is the hint of the presence of buried highly doped layers [9]. According to the literature, bipolar transistors in the differential pair found in the input stage core are very sensitive to charge deposition. However, experiments have shown that the SETs appear only using the highest energy value $(6 \mathrm{~nJ})$ and focusing on the most sensitive area $(270 \mu \mathrm{m})$. This result seems to disagree with the data from front-side laser irradiations. A possible explication is the presence of a buried highly doped Si layer, behaving like a metal, that absorbs most of the laser energy in such a way that only focusing in the most sensitive node with the highest laser intensity, enough energy is deposited to induce an SET.

Finally, the data support previous works that stated the high sensitivity of some of the transistors, such as Q9 and QR1. Moreover, data explore the sensitivity of these devices within the whole volume and not only in the Z-axis or the front surface.

\section{CONCLUSION}

We have pointed out that all layers of the chip can be reached by backside irradiation without previous thinning of the silicon bulk at a wavelength of $1300 \mathrm{~nm}$.

Backside irradiation using the two-photon absorption is an interesting method to find all the active layers inside an analog device as well as to detect the existence of shielding layers that cannot be traversed by a laser beam.

Sensitivity maps corresponding to different values of depth and energy are also shown in this paper, which can be associated with the equivalent ion hit.

In the future we shall focus our attention to fit the laser parameters to the ions to emulate

\section{ACKNOWLEDGMENT}

The authors would like to thank Dr. J. M. Miranda Pantoja, at the Departamento de Física Aplicada III of the Universidad Complutense de Madrid, for his support during the development of the data acquisition system. Also we wish to thank the Optics Department of the Universidad Complutense de Madrid.

\section{REFERENCES}

[1] S. Buchner, J. J. Howard, C. Poivey, D. McMorrow and R. Pease, "Pulsed-laser testing methodology for single event transients in linear devices", IEEE Transactions on Nuclear Science, Vol. 51, pp. 37163722, Dec. 2004.

[2] Sternberg, A. L.; Massengill, L. W.; Buchner, S.; Pease, R. L.; Boulghassoul, Y.; Savage, M. W.; McMorrow, D. and Weller, R. A. "The role of parasitic elements in the single-event transient response of linear circuits", IEEE Transactions on Nuclear Science, Vol. 49,pp. 31153120, Dec. 2002.

[3] Buchner, S.; McMorrow, D.; Poivey, C.; Howard, J. J.; Boulghassoul, Y.; Massengill, L. W.; Pease, R. \& Savage, M., "Comparison of singleevent transients induced in an operational amplifier (LM124) by pulsed laser light and a broad beam of heavy ions", IEEE Transactions on $\mathrm{Nu}$ clear Science, Vol. 51, pp. 2776- 2781, Dec. 2004.

[4] McMorrow, D.; Lotshaw, W. T.; Melinger, J. S.; Buchner, S.; Boulghassoul, Y.; Massengill, L. W. \& Pease, R. L., "Three-dimensional mapping of single-event effects using two photon absorption", IEEE Transactions on Nuclear Science, Vol. 50, pp. 2199-2207, Dec. 2003.

[5] McMorrow, D.; Lotshaw, W. T.; Melinger, J. S.; Buchner, S. \& Pease, R. L., "Subbandgap laser-induced single event effects: carrier generation via two-photon absorption", IEEE Transactions on Nuclear Science, Vol. 49, pp. 3002-3008, Dec. 2002.

[6] A. Akkerman and J. Barack, "Ion-track structure and its effects in small size volumes of silicon" IEEE Transactions on Nuclear Science, vol.49,pp.3022-3031, December 2002.

[7] R. Pease, "Modeling single event transients in bipolar linear circuits, "Nuclear Science, IEEE Transactions on Nuclear Science, vol. 55, no. 4, pp. 1879-1890, Aug. 2008.

[8] Savage, M.W.; Turflinger, T.; Titus, J.L.; Barsun, H.F.; Sternberg, A.; Boulghassoul, Y.; Massengill, L.W., "Variations in SET pulse shapes in the LM124A and LM111," Radiation Effects Data Workshop, pp. 75-81, 2002.

[9] D. Lewis et al., "Backside laser testing of ICs for SET sensitivity evaluation", IEEE Transactions on Nuclear Science, vol. 48, no. 6, p.2193, Dec. 2001 


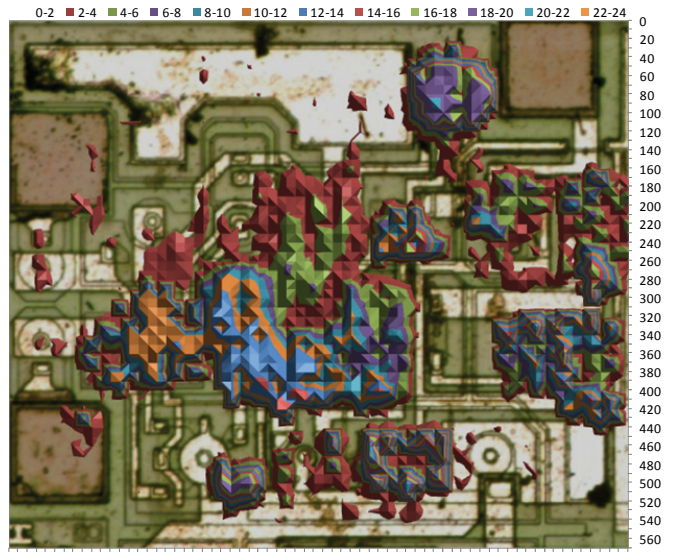

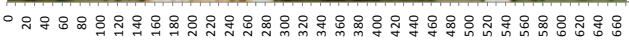

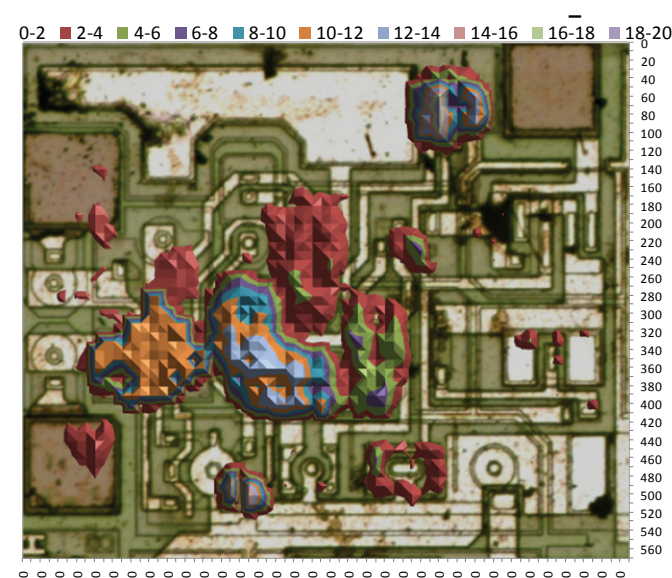

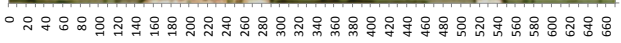

$0-2=2-4 \quad \square-6=6-8 \quad \square-10 \quad \square 10-12 \quad \square 12-14$

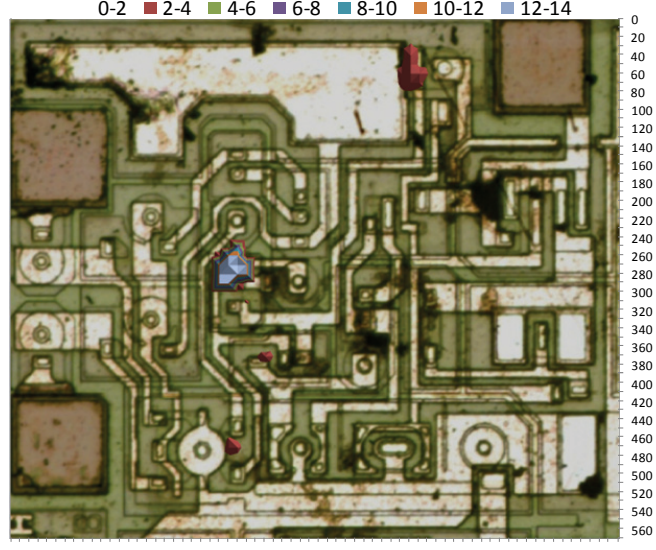

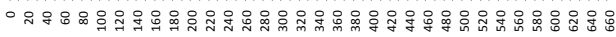

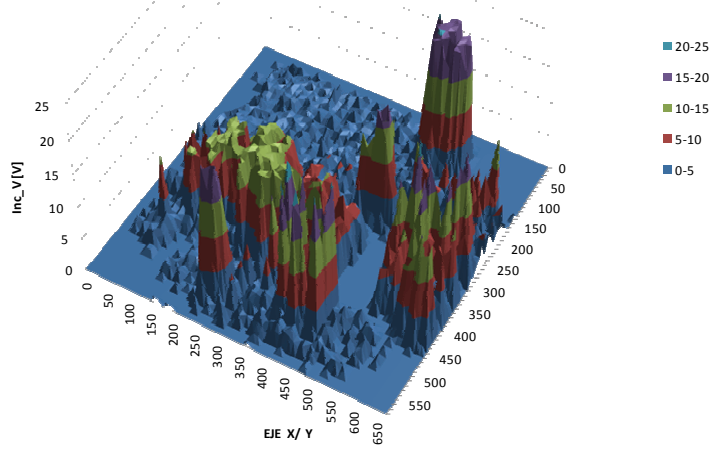

(a)

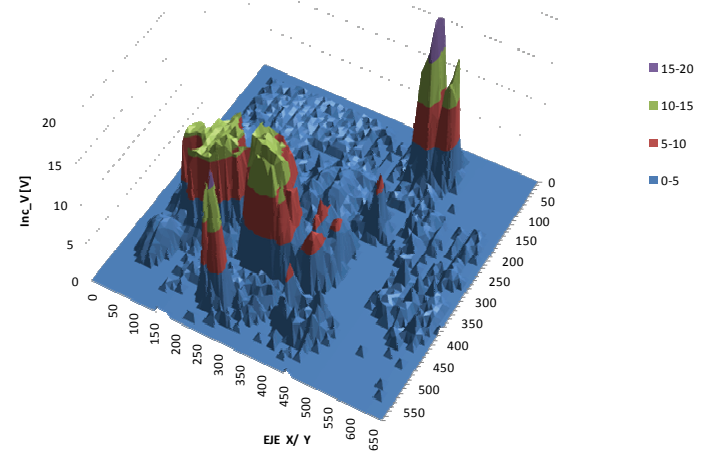

(b)

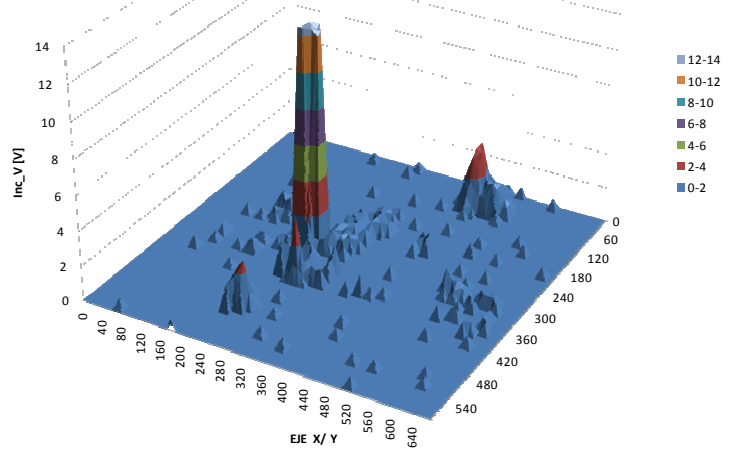

(c)

Fig. 11: XY Scans performed at a depth of $210 \mu \mathbf{m}$ using $6 \mathrm{~nJ}$ (a), $1.2 \mathrm{~nJ}(\mathrm{~b})$ and $0.6 \mathrm{~nJ}$ (c). Each color is related to the peak-peak voltage measured in the single event transient. All the lengths are expressed in $\mu \mathrm{m}$. 

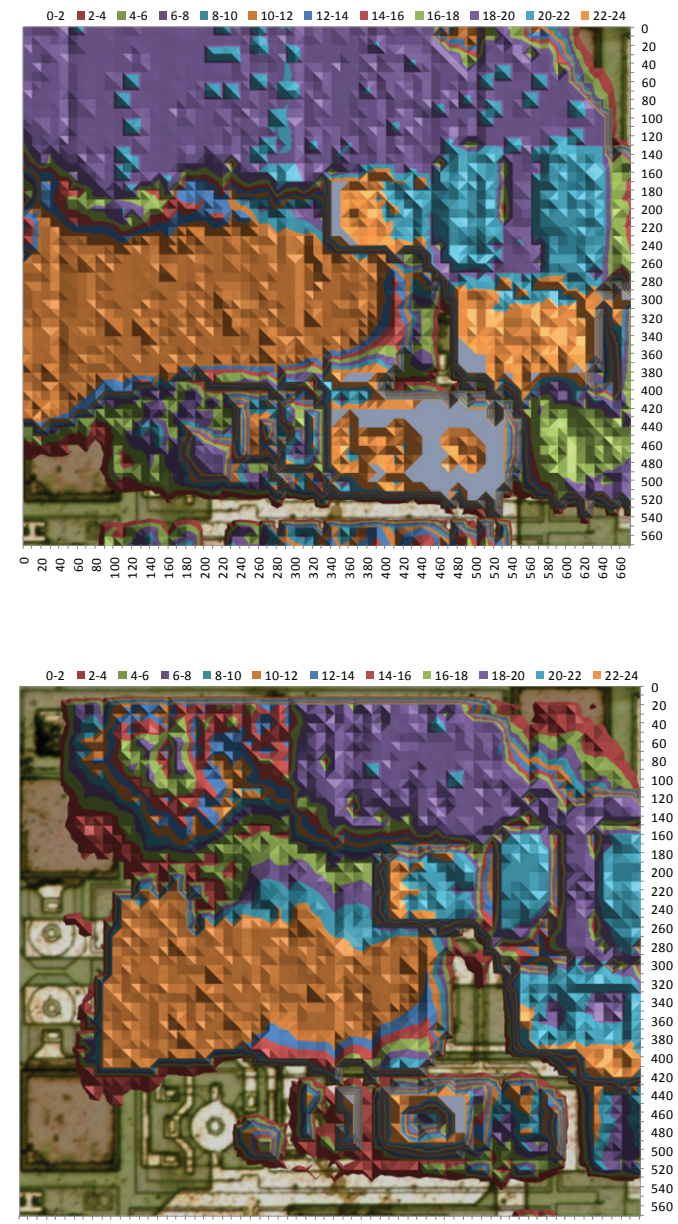

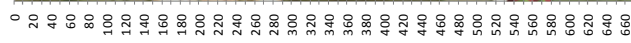

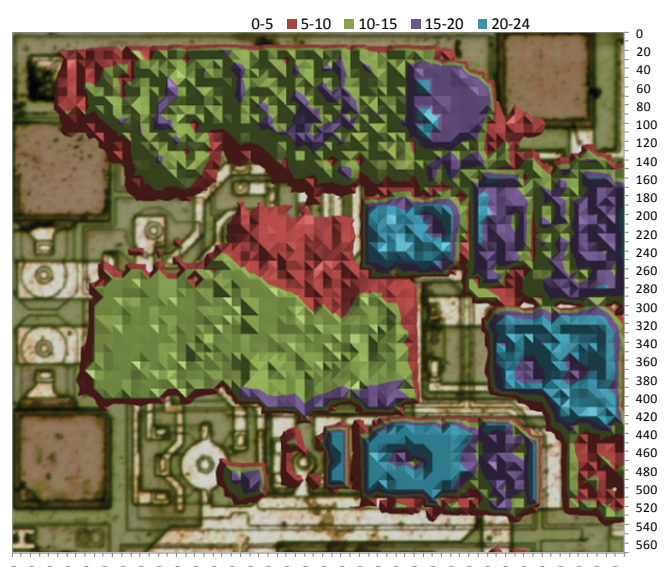

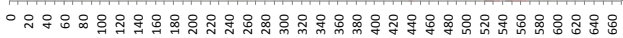

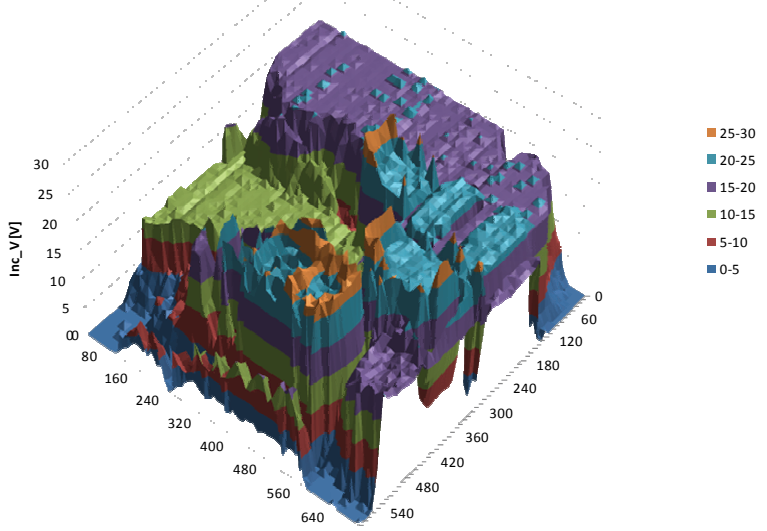

(a)

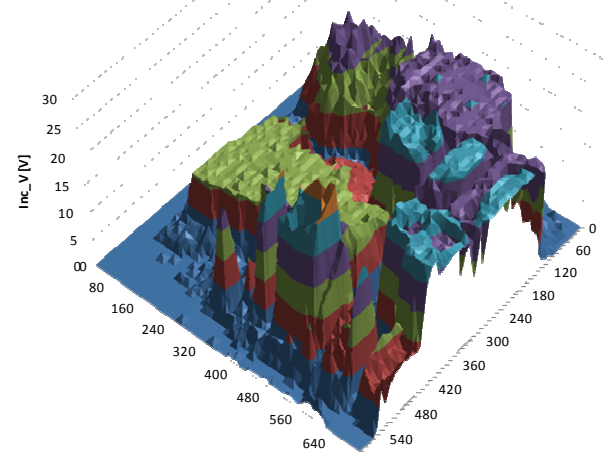

$=25-30$

$=20-25$

$=10-15$

- $5-10$

$=0-5$

(b)

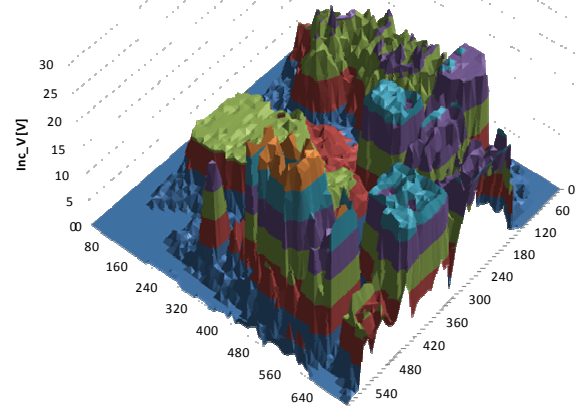

$=25-30$

$=20-25$

- $15-20$

- $5-10$

$=0-5$

(c)

Fig. 12: XY Scans performed at a depth of $270 \mu \mathrm{m}$ using $6 \mathrm{~nJ}$ (a), $1.2 \mathrm{~nJ}(\mathrm{~b})$ and $0.6 \mathrm{~nJ}(\mathrm{c})$. Each color is related to the peak-peak voltage measured in the single event transient. All the lengths are expressed in $\mu \mathrm{m}$. 